\title{
Chiral phosphoric acid and its applications of asymmetric catalytic hydrogenation
}

\author{
Sun Zhuoxin ${ }^{1 *}$ \\ ${ }^{1}$ School of Pharmaceutical Science and Technology, Tianjin University, Tianjin, 300072, China
}

\begin{abstract}
As an excellent representative of organic small molecular catalysts, chiral phosphoric acid played an important role in asymmetric catalytic hydrogenation. According to the skeleton of chiral phosphoric acid, the development and applications of chiral phosphoric acid were summarized. The applications of chiral phosphoric acid in asymmetric catalytic hydrogenation were introduced according to three kinds of hydrogen source systems used in chiral phosphoric acid catalytic reaction. It was found that chiral phosphoric acid has high activity and excellent selectivity. Chiral products with excellent yields and enantioselectivities were obtained in most reactions.
\end{abstract}

\section{Introduction}

In 2001, Professor Noyori, and Dr. Knowles won the Nobel Prize for their outstanding contributions in asymmetric catalytic hydrogenation. Since then, asymmetric catalytic hydrogenation of compounds has become a hot topic in the field of synthesis, and has been studied and reported by a large number of scientists and scholars. Asymmetric catalytic hydrogenation was first catalyzed by transition metals. Compared with small molecular catalysts, transition metal catalysts have the disadvantages of high toxicity, difficult to synthesize and recover. ${ }^{[1]}$ In 1989, Professor Batra first used amino acids as catalyst to realize asymmetric transfer hydrogenation without metal participation, which opened a new door for the asymmetric reduction of small molecular catalysts. ${ }^{[2]}$ Subsequently, the technology of small molecular catalysis has been developed rapidly, and the types of catalysts are becoming more and more abundant.

Chiral phosphoric acid is a kind of small molecular catalyst with excellent reaction activity and selectivity. It is divided into four types according to the difference of skeleton structures, which are: BINOL-type, $\mathrm{H}_{8}$-BINOL-type, SPINOL-type, VANOL-type and VAPOL-type (Figure 1) ${ }^{[3]}$

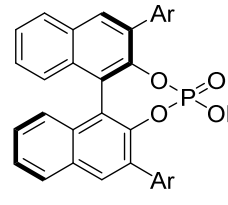

BINOL-PA

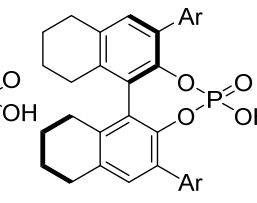

$\mathrm{H}_{8}$-BINOL-PA

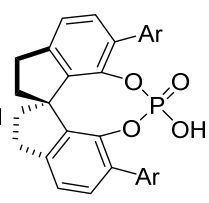

SPINOL-PA

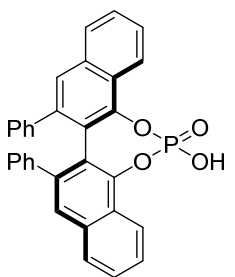

VANOL-PA

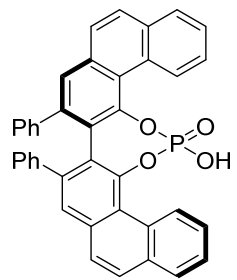

VAPOL-PA

Figure 1 Skeletons of chiral phosphoric acids.

Based on these skeletons, chiral phosphoric acids were further expanded by changing the types of 3,3'-substituents. With the further research, the catalytic mechanism of chiral phosphoric acid has been further understood. Taking BINOL-type phosphoric acid as an example: (1) chiral phosphoric acid has strong acidity, and can activate the substrate by forming ion pairs. (2) The oxygen in the double bond of oxygen and phosphorus can be used as Lewis base, so the chiral phosphoric acid can be regarded as a bifunctional catalyst. (3) By changing the substituents of R-group, different space types were constructed, and the direction of the combination of catalyst and reactant was changed, so as to improve the selectivity (Figure 2). ${ }^{[4]}$ 


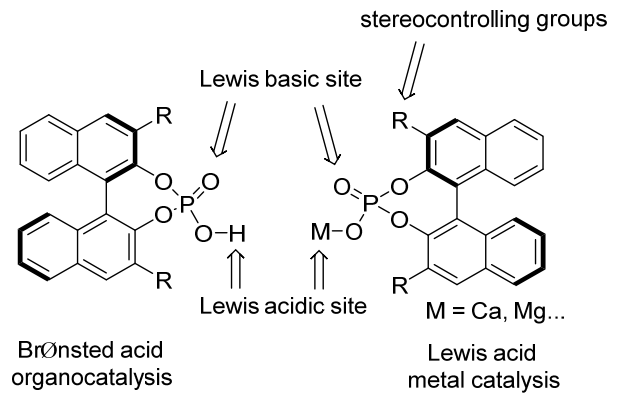

Figure 2 Potential of phosphoric acids and metal phosphates.

\section{Development of chiral phosphoric acid}

Although BINOL derived chiral phosphoric acids have been used in organic synthesis for nearly 50 years, their application as catalysts only started more than ten years ago. Akiyama and co-workers published a report on the Mannich reaction catalyzed by BINOL derived chiral phosphoric acid in 2004, which is widely considered as one of the pioneers in this field. ${ }^{[5]}$ Subsequently, other types of chiral phosphoric acids were gradually applied to asymmetric synthesis. Until 2006, Gong and co-workers first used $\mathrm{H}_{8}$-BINOL derived chiral phosphoric acid as catalyst to realize asymmetric Biginelli reaction, and obtained chiral products with excellent yields and enantioselectivities. ${ }^{[6]}$ In 2002, Zhou and co-workers used SPINOL as ligand, which was the first time that SPINOL was used in asymmetric synthesis. ${ }^{[7]}$ In 2010 , Wang and co-workers successfully synthesized SPINOL derived chiral phosphoric acid for the first time, and used it as a catalyst to efficiently realize the Friedel-Crafts reaction of indole and imine. ${ }^{[8]}$ The development of chiral phosphoric acid derived from VAPOL as catalyst in asymmetric synthesis was also very dramatic. In 2005, Antilla and co-workers successfully synthesized the chiral phosphoric acid with VAPOL for the first time, and used it as a catalyst for reductive amination to obtain chiral amines with excellent yields and enantioselectivities. ${ }^{\left[{ }^{9]}\right.}$ However, because the catalytic mechanism of chiral phosphoric acid was not fully understood at that time, we mistakenly thought that the chiral phosphoric acid derived from VAPOL was an effective catalyst in the reaction. Until 2013, Sala revealed that the real catalyst in the reaction should be chiral phosphate through a series of complex control experiments. ${ }^{[10]}$ Subsequently, chiral phosphates derived from VAPOL have been well developed as catalysts for asymmetric reactions.

\section{Asymmetric reduction catalyzed by chiral phosphoric acid}

There are three kinds of hydrogen sources used in asymmetric transfer hydrogenation of chiral phosphoric acid: Hantzsch ester, benzothiazoline and borane. Hantzsch esters were widely used in asymmetric transfer hydrogenation catalyzed by chiral phosphoric acid.

In 2005, Reuping and co-workers first reported the asymmetric reduction of imines using chiral phosphoric acid as catalyst and Hantzsch ester as hydrogen source. ${ }^{[1]}$ The chiral amines with excellent yields and enantioselectivities (Figure 3) were obtained by the reduction system. Since then, asymmetric reduction based on the system has been reported, including asymmetric reduction of nitrogen heterocyclic derivatives with high reduction difficulty.

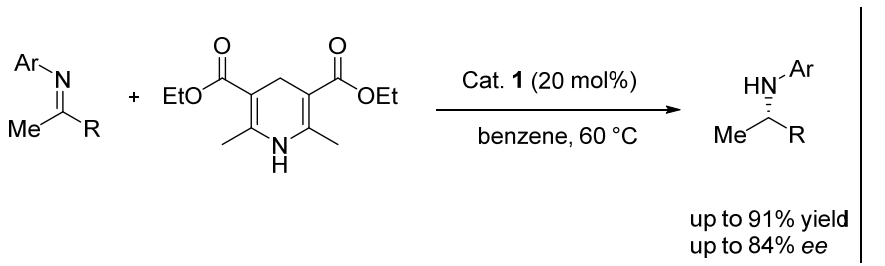

Figure 3 Asymmetric transfer hydrogenation of imines.

Benzothiazoline as a hydrogen source has also been shown in asymmetric reduction catalyzed by chiral phosphoric acid. In 2009, Akiyama and co-workers first used chiral phosphoric acid as catalyst and benzothiazoline as hydrogen source to asymmetric reduction of ketimides and obtained chiral products with excellent yields and enantioselectivities (Figure 4). ${ }^{[12]}$ Then, asymmetric reduction reaction based on this reduction system has been widely used. They proposed that the reaction accorded with the bifunctional mechanism of coordination between benzothiazoline and phosphoric acid's Lewis basic site. 

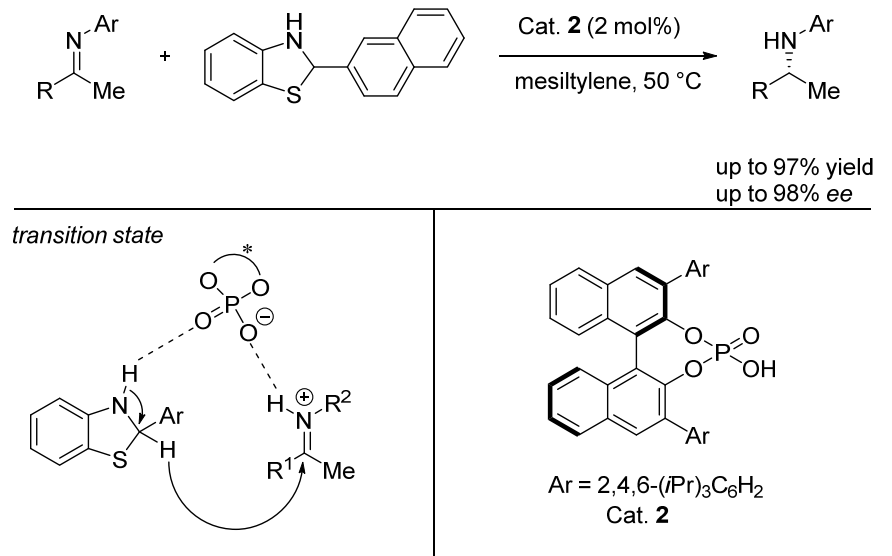

Figure 4 Asymmetric transfer hydrogenation with benzothiazoline of imines.

In the present reports, the asymmetric reduction reactions of Hantzsch esters or benzothiazolines as hydrogen sources mostly act on imines or imines, but there are few reports on the reduction of ketones. In 2011, Antilla and co-workers first used borane and chiral phosphoric acid to achieve asymmetric reduction of aromatic ketones, and obtained chiral products with excellent yields and enantioselectivities (Figure 5). ${ }^{[13]}$ At the same time, a combined with borane to form a kind of chiral boro-phosphate catalyst in situ. The catalyst was a kind of bifunctional catalyst. Lewis acid in the boron center could activate ketones, and the oxygen in $\mathrm{P}=\mathrm{O}$ could be used as Lewis base to improve the affinity of borane. At the same time, the hydrogen in unreacted borane attacked the activated ketones to form chiral products possible reaction mechanism was proposed. It was speculated that in this reaction, chiral phosphoric acid

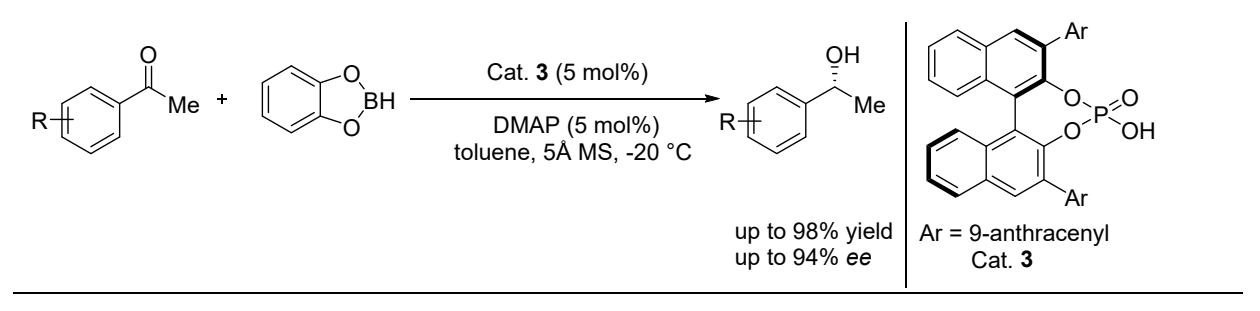

transition state
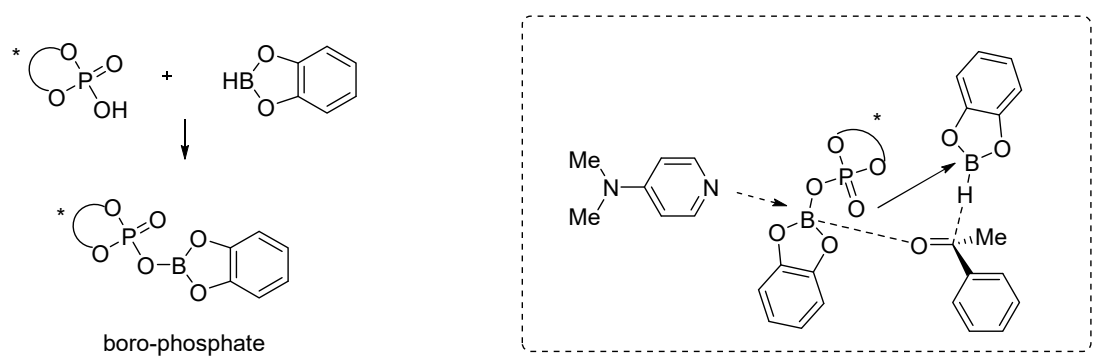

Figure 5 Asymmetric transfer hydrogenation with borane of ketones.

\section{Conclusion}

Chiral phosphoric acid is one of the outstanding representatives of organic small molecular catalysts. Compared with transition metals, chiral phosphoric acid has the advantages of low toxicity and easy synthesis and recovery, which provides a new idea for the synthesis of a variety of compounds with pharmacological activities. These catalysts performed well in asymmetric catalytic hydrogenation. Most of the chiral products obtained in asymmetric transfer hydrogenation have excellent yields and high ee values. However, it should also be noted that most of the reports have not reached the gram level reaction due to the limitations of the current catalytic reaction, and there are still many limitations in the realization of industrial production.

\section{Acknowledgments}

I would like to thank my supervisor for giving me a better understanding of the field of chiral phosphoric acid catalysis. I would also like to thank my team members for their help and my family for their support. 


\section{References}

1. P. I. Dalko, L. Moisan, Angew. Chem., Int. Ed. 43, 5138 (2004)

2. S. Singh, U. K. Batra, Indian J. Chem., Sect. B: Org. Chem. Incl. Med. Chem. 28, 1 (1989)

3. D. Parmar, E. Sugiono, S. Raja, M. Rueping, Chem. Rev. 117, 10608 (2017)

4. M. Rueping, E. Sugiono, F. R. Schoepke, Synlett 6, 852 (2010)

5. T. Akiyama, J. Itoh, K. Yokota, K. Fuchibe, Angew. Chem., Int. Ed. 43, 1566 (2004)

6. X. Chen, X. Xu, L. Hua, L. Cun, L. Gong, J. Am. Chem. Soc. 128, 14802 (2006)
7. Y. Fu, J. H. Xie, A. L. Hu, H. Zhou, L.-X. Wang, Q.-L. Zhou, Chem. Commun. 5, 480 (2002)

8. F. Xu, D. Huang, C. Han, W. Shen, X. Lin, Y. Wang, J. Org. Chem. 75, 8677 (2010)

9. G. B. Rowland, H. Zhang, E. B. Rowland, S. Chennamadhavuni, Y. Wang, J. C. Antilla, J. Am. Chem. Soc. 127, 15696 (2005)

10. G. D. Sala, Tetrahedron 69, 50 (2013)

11. T. Akiyama, Chem. Rev. 107, 5744 (2007)

12. C. Zhu, T. Akiyama, Org. Lett. 11, 4180 (2009)

13. Z. Zhang, P. Jain, J. C. Antilla, Angew. Chem., Int. Ed. 123, 11153 (2011) 